\title{
Dynamic Behaviors of Holling Type II Predator-Prey System with Mutual Interference and Impulses
}

\author{
Hongli Li, Long Zhang, Zhidong Teng, and Yaolin Jiang \\ College of Mathematics and System Sciences, Xinjiang University, Urumqi 830046, China \\ Correspondence should be addressed to Long Zhang; longzhang_xj@sohu.com
}

Received 22 November 2013; Accepted 30 April 2014; Published 29 May 2014

Academic Editor: Guang Zhang

Copyright (C) 2014 Hongli Li et al. This is an open access article distributed under the Creative Commons Attribution License, which permits unrestricted use, distribution, and reproduction in any medium, provided the original work is properly cited.

\begin{abstract}
A class of Holling type II predator-prey systems with mutual interference and impulses is presented. Sufficient conditions for the permanence, extinction, and global attractivity of system are obtained. The existence and uniqueness of positive periodic solution are also established. Numerical simulations are carried out to illustrate the theoretical results. Meanwhile, they indicate that dynamics of species are very sensitive with the period matching between species' intrinsic disciplinarians and the perturbations from the variable environment. If the periods between individual growth and impulse perturbations match well, then the dynamics of species periodically change. If they mismatch each other, the dynamics differ from period to period until there is chaos.
\end{abstract}

\section{Introduction}

As pointed out by Berryman [1], the dynamic relationship between predators and their prey has long been and will continue to be one of the dominant themes in both biology and mathematical biology due its universal existence and importance. Though predator-prey models with periodic or almost periodic coefficients have been studied extensively (see [2-14] and the references cited therein), few papers consider the mutual interference between the predators and prey, which was introduced by Hassell in 1971. During his research of the capturing behavior between hosts and parasites, he found that the hosts or parasites had the tendency to leave each other when they met, which interfered with hosts capture effects. Obviously, the mutual interference will be stronger, while the size of parasite becomes larger. From the observation, Hassell introduced the concept of mutual interference constant $m(0<m \leq 1)$ and established a Lotka-Volterra predator-prey model with mutual interference as follows:

$$
\begin{array}{r}
\dot{x}(t)=x g(x)-\varphi(x) y^{m}, \\
\dot{y}(t)=y\left(-d+\beta \varphi(x) y^{m-1}-q(y)\right),
\end{array}
$$

where $x$ and $y$ are the sizes of the prey and predator populations, respectively, and $m$ is mutual interference constant; see
$[15,16]$ for more details on the biological meaning of mutual interference constant $m$. Some results have appeared for this type of predator-prey system [17-20].

Recently, much more attention has paid on the functional responses. Based on experiments, Holling [21] investigated three different kinds of functional responses for different kinds of species to model the phenomena of predation, which made the standard Lotka-Volterra system more consistent with the real ecosystem. The Lotka-Volterra system with Holling type II functional response is introduced by Wang and Zhu [22] in the following form:

$$
\begin{aligned}
& \dot{x}(t)=x(t)\left(r_{1}(t)-b_{1}(t) x(t)\right)-\frac{c_{1}(t) x(t)}{k+x(t)} y^{m}(t), \\
& \dot{y}(t)=y(t)\left(-r_{2}(t)-b_{2}(t) y(t)\right)+\frac{c_{2}(t) x(t)}{k+x(t)} y^{m}(t) .
\end{aligned}
$$

However, ecological system is often deeply perturbed by nature and human exploit activities (e.g., fire, drought, flooding deforestation, hunting, harvesting, breeding, etc.), which are not suitable to be considered continually. To accurately describe the system, impulsive differential equations may be a better candidate than ordinary differential equation. The theory of impulsive differential equations not only is now being recognized to be richer than the corresponding theory 
TABle 1: Parameter values used in the simulations of system (3).

\begin{tabular}{lcc}
\hline Parameter & Interpretation & Value \\
\hline$r_{1}(t)$ & Growth rate of the prey & $1+\sin (2 \pi t)$ \\
$r_{2}(t)$ & Death rate of the predator & $0.03+0.01 \sin (2 \pi t)$ \\
$b_{1}(t)$ & Intracompetition coefficient among the prey & $1-0.1 \sin (2 \pi \mathrm{t})$ \\
$b_{2}(t)$ & Intracompetition coefficient among the predators & $0.4-0.1 \sin (2 \pi t)$ \\
$c_{1}(t)$ & Capture rate of predator on prey & $0.7+0.1 \cos (2 \pi t)$ \\
$c_{2}(t)$ & Conversion rate of nutrition & $0.2+0.1 \sin (2 \pi t)$ \\
$k$ & The environment carrying capacity & 10 \\
$m$ & Mutual interference constant & $1 / 3$ \\
$h_{k}$ & Impulse perturbations on prey & - \\
$g_{k}$ & Impulse perturbations on predator & - \\
\hline
\end{tabular}

of differential equations without impulse, but also represents a more natural framework for mathematical modelling of many real-world phenomena. In recent years, many important and interesting results on the permanence, extinction, global attractivity of systems, the existence and uniqueness of positive periodic solutions, bifurcation and dynamical complexity, and so forth have been proposed in [23-28] and references cited therein. Motivated by above studies, in this paper we will consider the following Holling II predator-prey system with mutual interference and impulses:

$$
\begin{array}{r}
\dot{x}(t)=x(t)\left(r_{1}(t)-b_{1}(t) x(t)\right)-\frac{c_{1}(t) x(t)}{k+x(t)} y^{m}(t), \\
\dot{y}(t)=y(t)\left(-r_{2}(t)-b_{2}(t) y(t)\right)+\frac{c_{2}(t) x(t)}{k+x(t)} y^{m}(t), \\
t \neq \tau_{k}, \\
x\left(\tau_{k}^{+}\right)=\left(1+h_{k}\right) x\left(\tau_{k}\right), \\
y\left(\tau_{k}^{+}\right)=\left(1+g_{k}\right) y\left(\tau_{k}\right), \\
t=\tau_{k}, k=1,2, \ldots,
\end{array}
$$

where $x$ and $y$ are the sizes of the prey and predator populations, respectively. $0<m<1$ and $k$ is a positive constant. $r_{i}(t), b_{i}(t)$, and $c_{i}(t)(i=1,2)$ are continuous periodic functions defined on $[0,+\infty)$ with a common period $T>0 . b_{i}(t), c_{i}(t),(i=1,2)$ are strictly positive. Also $\tau_{k} \rightarrow$ $+\infty(t \rightarrow+\infty), 0<\tau_{1}<\tau_{2}<\tau_{3}<\cdots<\tau_{k}<\tau_{k+1}<\cdots$. Assume that $h_{k}, g_{k}(k=1,2 \ldots)$ are constants. $1+h_{k}>0$ and $1+g_{k}>0$, and there exists an integer $q>0$ such that $h_{k+q}=h_{k}, g_{k+q}=g_{k}, \tau_{k+q}=\tau_{k}+T$. The interpretations of parameters can be seen in Table 1 .

The organization of this paper is as follows. In Section 2, we give some definitions and state some lemmas which are essential to study the main theorems. In Section 3, sufficient conditions for the permanence, extinction of system (3), and the existence, uniqueness, and global attractivity of positive periodic solution are obtained. In Section 4, numerical examples are presented to illustrate our results. In the last section, we give a brief discussion.

\section{Preliminaries}

In this section, we introduce some notations and definitions and state some lemmas which will be useful in the subsequent sections.

Let $R_{+}=[0, \infty), R_{+}^{2}=\left\{X \in R^{2} \mid X \geq 0\right\}$. The map $f=$ $\left(f_{1}, f_{2}\right)^{T}$ is defined by the right hand of the first and second equations of system (3). Let $V: R_{+} \times R_{+}^{2} \rightarrow R_{+}$; then $V$ is said to belong to class $V_{0}$ if

(1) $V$ is continuous in $\left(\tau_{k}, \tau_{k+1}\right] \times R_{+}^{2}$ and for each $X \in$ $R_{+}^{2}, k=1,2, \ldots$, there exists $\lim _{(t, Y) \rightarrow\left(\tau_{k}^{+}, X\right)} V(t, Y)=$ $V\left(\tau_{k}^{+}, X\right)$;

(2) $V$ is locally Lipschitzian in $X$.

Definition 1. Let $V \in V_{0}$; then, for $(t, X) \in\left(\tau_{k}, \tau_{k+1}\right] \times R_{+}^{2}$, the upper right derivative of $V(t, X)$ with respect to the impulsive differential system (3) is defined as

$$
\begin{aligned}
& D^{+} V(t, x) \\
& \quad=\lim _{h \rightarrow 0^{+}} \sup \frac{1}{h}[V(t+h, X+h f(t, X))-V(t, X)] .
\end{aligned}
$$

Definition 2. System (3) is permanent, if, for any positive solution $(x(t), y(t))^{T}$ of system (3), there exist positive constants $m_{1}, m_{2}, M_{1}$, and $M_{2}$ such that

$$
\begin{aligned}
& m_{1} \leq \liminf _{t \rightarrow+\infty} x(t) \leq \limsup _{t \rightarrow+\infty} x(t) \leq M_{1}, \\
& m_{2} \leq \liminf _{t \rightarrow+\infty} y(t) \leq \limsup _{t \rightarrow+\infty} y(t) \leq M_{2} .
\end{aligned}
$$

Lemma 3 ([29]). Let $V \in V_{0}$ and $X(t)$ is a solution of (3) with initial value $X\left(0^{+}\right)=X_{0}$. Assume that

$$
\begin{gathered}
D^{+} V(t, X(t)) \leq g(t, V(t, X(t))), \quad t \neq \tau_{k}, k=1,2, \ldots, \\
V\left(t, X\left(t^{+}\right)\right) \leq \psi_{k}(V(t, X(t))), \quad t=\tau_{k}, k=1,2, \ldots,
\end{gathered}
$$

where $g: R_{+} \times R_{+} \rightarrow R$ is continuous in $\left(\tau_{k}, \tau_{k+1}\right] \times R_{+}$and for $u \in R_{+}, k=1,2, \ldots, \lim _{(t, v) \rightarrow\left(\tau_{k}^{+}, u\right)} g(t, v)=g\left(\tau_{k}^{+}, u\right)$ exists 
and $\psi_{k}: R_{+} \rightarrow R_{+}$is nondecreasing. Let $r(t)$ be the maximal solution of scalar impulsive differential equation

$$
\begin{array}{r}
\dot{u}(t)=g(t, u(t)), \quad t \neq \tau_{k}, \quad k=1,2, \ldots, \\
u\left(t^{+}\right)=\psi_{k}(u(t)) \geq 0, \quad t=\tau_{k}, t_{k}>0, \quad k=1,2, \ldots, \\
u\left(0^{+}\right)=u_{0}
\end{array}
$$

existing on $[0, \infty)$. Then $V\left(0^{+}, X_{0}\right) \leq u_{0}$ implies that $V(t, X(t)) \leq r(t)$ for all $t \geq 0$.

Similarly, assume that inequality (6) is reversed. Let $p(t)$ be the minimal solution of (7) existing on $[0,+\infty)$. Then, $V\left(0^{+}, X_{0}\right) \geq u_{0}$ implies that $V(t, X(t)) \geq p(t)$ for all $t \geq 0$.

Let $f(t)$ be continuous $T$-periodic function defined on the $R_{+}$; we introduce the following notation:

$$
m[f(t)]=\frac{1}{T} \int_{0}^{T} f(t) d t
$$

Consider the following periodic logistic equation with impulses:

$$
\begin{aligned}
\dot{x}(t) & =x(t)\left(r_{1}(t)-b_{1}(t) x(t)\right), \quad t \neq \tau_{k}, \\
x\left(\tau_{k}^{+}\right) & =\left(1+h_{k}\right) x\left(\tau_{k}\right), \quad t=\tau_{k}, k=1,2, \ldots
\end{aligned}
$$

We have the following results.

Lemma 4 (see [30]). (1) If

$$
\sum_{k=1}^{q} \ln \left(1+h_{k}\right)+\operatorname{Tm}\left[r_{1}(t)\right]>0
$$

then system (9) has a unique T-periodic solution $x^{*}(t)$, which is globally asymptotically stable.

(2) If

$$
\sum_{k=1}^{q} \ln \left(1+h_{k}\right)+\operatorname{Tm}\left[r_{1}(t)\right] \leq 0
$$

then $x^{*}(t) \equiv 0$.

Consider a periodic Logistic equation with impulses:

$$
\begin{aligned}
\dot{x}(t) & =x(t)\left(b(t)-a(t)(x(t))^{\theta}\right), \quad t \neq \tau_{k}, \\
x\left(\tau_{k}^{+}\right) & =\left(1+h_{k}^{\prime}\right) x\left(\tau_{k}\right), \quad t=\tau_{k}, \quad k=1,2, \ldots,
\end{aligned}
$$

where $\theta$ is a positive constant, $a(t)$ and $b(t)$ are continuous Tperiodic functions with $a(t)>0$ and $m[b(t)]>0$, and $h_{k+q}^{\prime}=$ $h_{k}^{\prime}, \tau_{k+q}=\tau_{k}+T$. We have the following results.

Lemma 5 (see [31]). (1) If

$$
\sum_{k=1}^{q} \ln \left(1+h_{k}^{\prime}\right)+\operatorname{Tm}[b]>0,
$$

then system (10) has a unique T-periodic solution $\widetilde{x^{*}}(t)$, which is globally asymptotically stable.
(2) If

$$
\sum_{k=1}^{q} \ln \left(1+h_{k}^{\prime}\right)+\operatorname{Tm}[b(t)]<0
$$

then $\widetilde{x^{*}}(t) \equiv 0$.

Lemma 6 (see [31]). Let $x^{*}(t)$ be the unique T-periodic positive solution of system (9) and $\widetilde{x_{\varepsilon}^{*}}(t)$ the unique T-periodic positive solution of the following system:

$$
\begin{gathered}
\dot{x}(t)=x(t)\left(b_{\varepsilon}(t)-a(t)(x(t))^{\theta}\right), \quad t \neq \tau_{k}, \\
x\left(\tau_{k}^{+}\right)=\left(1+h_{k}^{\prime}\right) x\left(\tau_{k}\right), \quad t=\tau_{k}, k=1,2, \ldots,
\end{gathered}
$$

where $b_{\varepsilon}(t)$ is the continuous T-periodic function with $m\left[b_{\varepsilon}(t)\right]>0$ and $\lim _{\varepsilon \rightarrow 0} b_{\varepsilon}(t)=b(t)$. Then

$$
\lim _{\varepsilon \rightarrow 0}\left|\widetilde{x_{\varepsilon}^{*}}(t)-x^{*}(t)\right|=0 \text {. }
$$

Furthermore, we consider the following periodic logistic equation with impulses:

$$
\begin{array}{r}
\dot{v}(t)=v(t)\left(r_{2}(t)-c_{2}(t) \frac{x^{*}(t)}{k+x^{*}(t)} v^{1-m}(t)\right), \quad t \neq \tau_{k}, \\
v\left(\tau_{k}^{+}\right)=\frac{1}{1+g_{k}} v\left(\tau_{k}\right), \quad t=\tau_{k}, \quad k=1,2, \ldots
\end{array}
$$

By Lemma 5, we have the following Corollary.

Corollary 7. If

$$
-\sum_{k=1}^{q} \ln \left(1+g_{k}\right)+\operatorname{Tm}\left[r_{2}(t)\right]>0,
$$

then system (15) has a unique T-periodic solution $\widetilde{v^{*}}(t)$, which is globally asymptotically stable.

Next, one considers the following periodic logistic equation with impulses:

$$
\begin{array}{r}
\dot{x}(t)=x(t)\left(r_{1}(t)-\frac{c_{1}(t)}{k} \widetilde{y^{*}}(t)^{m}-b_{1}(t) x(t)\right), \quad t \neq \tau_{k}, \\
x\left(\tau_{k}^{+}\right)=\left(1+h_{k}\right) x\left(\tau_{k}\right), \quad t=\tau_{k}, \quad k=1,2, \ldots .
\end{array}
$$

By Lemma 5, we have the following Corollary.

Corollary 8. If

$$
\sum_{k=1}^{q} \ln \left(1+h_{k}\right)+\operatorname{Tm}\left[r_{1}(t)-\frac{c_{1}(t)}{k} \widetilde{y^{*}}(t)^{m}\right]>0,
$$

then system (16) has a unique T-periodic solution $x_{*}(t)$, which is globally asymptotically stable. 


\section{Main Results}

In this section, we will study the permanence, extinction, and global attractivity of system (3) and the existence and uniqueness of positive periodic solution.

Lemma 9. For any solution $(x(t), y(t))^{T}$ of system (3), if $x\left(0^{+}\right)>0, y\left(0^{+}\right)>0$, then $x(t)>0$ and $y(t)>0$ for all $t \geq 0$.

Proof. From the first equation of (3), one has

$$
\dot{x}(t)=p(t) x(t), \quad t \neq \tau_{k},
$$

where

$$
p(t)=r_{1}(t)-b_{1}(t) x(t)-\frac{c_{1}(t)}{k+x(t)} y^{m}(t) ;
$$

thus, from $x\left(0^{+}\right)>0$, we have

$$
x(t)=\prod_{0<\tau_{k}<t}\left(1+h_{k}\right) x\left(0^{+}\right) \exp \left(\int_{0}^{t} p(s) d s\right)>0 .
$$

Similarly, it follows from the second equation of system (3) that $y(t)>0$. This completes the proof of Lemma 9 .

Lemma 10. Assuming that $\left(A_{1}\right)$ and $\left(H_{1}\right)$ hold, for any positive solution $(x(t), y(t))^{T}$ of system (3), there exist constants $M_{i}(i=1,2)$ such that

$$
\limsup _{t \rightarrow+\infty} x(t) \leq M_{1}, \quad \limsup _{t \rightarrow+\infty} y(t) \leq M_{2} .
$$

Proof. First, we show that $x(t)$ is ultimate upper bounded. It follows from (3) that

$$
\begin{aligned}
\dot{x}(t) & \leq x(t)\left(r_{1}(t)-b_{1}(t) x(t)\right), \quad t \neq \tau_{k}, \\
x\left(\tau_{k}^{+}\right) & =\left(1+h_{k}\right) x\left(\tau_{k}\right), \quad t=\tau_{k}, \quad k=1,2, \ldots .
\end{aligned}
$$

Consider the following auxiliary system:

$$
\begin{aligned}
\dot{z}(t) & =z(t)\left(r_{1}(t)-b_{1}(t) z(t)\right), \quad t \neq \tau_{k}, \\
z\left(\tau_{k}^{+}\right) & =\left(1+h_{k}\right) z\left(\tau_{k}\right), \quad t=\tau_{k}, \quad k=1,2, \ldots
\end{aligned}
$$

By Lemma 3, we have $x(t) \leq z(t)$, where $z(t)$ is the solution of (22) with $z\left(0^{+}\right)=x\left(0^{+}\right)$. By $\left(\mathrm{A}_{1}\right)$, from Lemma 4 we get that system (22) has a unique $T$-periodic solution $x^{*}(t)$, which is globally stable. Therefore, for any $\varepsilon>0$ being small enough, there exists a $T_{1}>0$ such that

$$
\left|z(t)-x^{*}(t)\right|<\varepsilon
$$

for any $t>T_{1}$. Let $M_{1}=\sup \left\{x^{*}(t): t \in[0, T]\right\}$; we have

$$
x(t) \leq z(t)<x^{*}(t)+\varepsilon \leq M_{1}+\varepsilon
$$

for any $t>T_{1}$. Let $\varepsilon \rightarrow 0$; we obtain

$$
\limsup _{t \rightarrow+\infty} x(t) \leq M_{1} \text {. }
$$

Next, we prove that $y(t)$ is ultimately upper bounded. Define $y(t)=1 / u(t)$. From system (3), we have

$$
\begin{gathered}
\dot{u}(t)=u(t)\left(r_{2}(t)+b_{2}(t) \frac{1}{u(t)}\right. \\
\left.\quad-c_{2}(t) \frac{x(t)}{k+x(t)} u^{1-m}(t)\right), \quad t \neq \tau_{k}, \\
u\left(\tau_{k}^{+}\right)=\frac{1}{1+g_{k}} u\left(\tau_{k}\right), \quad t=\tau_{k}, k=1,2, \ldots
\end{gathered}
$$

For any $t>T_{1}$ and $t \neq \tau_{k}(k=1,2, \ldots)$, we have

$$
\begin{aligned}
\dot{u}(t) & \geq u(t)\left(r_{2}(t)-c_{2}(t) \frac{x(t)}{k+x(t)} u^{1-m}(t)\right) \\
& \geq u(t)\left(r_{2}(t)-c_{2}(t) \frac{x^{*}(t)+\varepsilon}{k+x^{*}(t)+\varepsilon} u^{1-m}(t)\right) .
\end{aligned}
$$

Consider the following auxiliary system:

$$
\begin{gathered}
\dot{\psi}(t)=\psi(t)\left(r_{2}(t)-c_{2}(t) \frac{x^{*}(t)+\varepsilon}{k+x^{*}(t)+\varepsilon} \psi^{1-m}(t)\right), \\
t \neq \tau_{k}, \\
\psi\left(\tau_{k}^{+}\right)=\frac{1}{1+g_{k}} \psi\left(\tau_{k}\right), \quad t=\tau_{k}, k=1,2, \ldots
\end{gathered}
$$

By Lemma 3, for any $t>T_{1}$, we have $u(t) \geq \psi(t)$, where $\psi(t)$ is the solution of (28) with $\psi\left(T^{+}\right)=u\left(T^{+}\right)$. By $\left(\mathrm{H}_{1}\right)$, from Lemma 5, system (28) has a unique $T$-periodic solution $\widetilde{v_{\varepsilon}^{*}}(t)$, which is globally stable. Therefore, letting $\varepsilon \rightarrow 0$, from Lemma 6 , we have $\widetilde{v_{\varepsilon}^{*}}(t) \rightarrow \widetilde{v^{*}}(t)$. Letting $M_{2}^{*}=\inf \left\{v^{*}(t):\right.$ $t \in[0, T]\}$, for any $\varepsilon_{0}>0$ being small enough, there exists $T_{2}>T_{1}$, and we have

$$
u(t)=\frac{1}{y(t)} \geq \psi(t)>\widetilde{v^{*}}(t)-\varepsilon_{0} \geq M_{2}^{*}-\varepsilon_{0}
$$

for any $t>T_{2}$. Let $\varepsilon_{0} \rightarrow 0$; we have

$$
\limsup _{t \rightarrow+\infty} y(t) \leq \frac{1}{M_{2}^{*}} \triangleq M_{2} \text {. }
$$

This completes the proof of Lemma 10.

Theorem 11. Assume that $\left(H_{1}\right)$ and $\left(H_{2}\right)$ hold, and

$$
\begin{gathered}
\sum_{k=1}^{q} \ln \left(1+g_{k}\right)+\operatorname{Tm}\left[-r_{2}(t)+\frac{c_{2}(t) x_{*}(t)}{k+x_{*}(t)} \widetilde{y^{*}}(t)^{m-1}\right]>0, \\
m\left[r_{1}(t)-\frac{c_{1}(t)}{k}\left(\widetilde{y^{*}}(t)\right)^{m}\right]>0, \\
m\left[-r_{2}(t)+\frac{c_{2}(t) x_{*}(t)}{k+x_{*}(t)}\left(\widetilde{y^{*}}(t)\right)^{m-1}\right]>0,
\end{gathered}
$$

where $x_{*}(t)$ is the unique T-periodic solution of system (16) and $\widetilde{y^{*}}(t) \triangleq 1 / \widetilde{v^{*}}(t)$. Then species $x$ and $y$ are permanent. 
Proof. From Lemma 9, there exist constants $M_{i}(i=1,2)$ such that

$$
\limsup _{t \rightarrow+\infty} x(t) \leq M_{1}, \quad \limsup _{t \rightarrow+\infty} y(t) \leq M_{2}, \quad i=1,2 .
$$

From the proof of Lemma 10, for above $\varepsilon>0$ being small enough, there exists $T_{2}>0$ such that

$$
y(t) \leq \frac{1}{\widetilde{v^{*}}(t)-\varepsilon} \triangleq \widetilde{y_{\varepsilon}^{*}}(t)
$$

for any $t>T_{2}$. From the first equation of system (3), for $t>T_{2}$ and $t \neq \tau_{k}$, we have

$$
\begin{aligned}
\dot{x}(t) & \geq x(t)\left(r_{1}(t)-\frac{c_{1}(t)}{k+x(t)} \widetilde{y_{\varepsilon}^{*}}(t)^{m}-b_{1}(t) x(t)\right), \\
& \geq x(t)\left(r_{1}(t)-\frac{c_{1}(t)}{k} \widetilde{y_{\varepsilon}^{*}}(t)^{m}-b_{1}(t) x(t)\right) .
\end{aligned}
$$

Consider the following auxiliary system:

$$
\begin{gathered}
\dot{\phi}(t)=\phi(t)\left(r_{1}(t)-\frac{c_{1}(t)}{k} \widetilde{y_{\varepsilon}^{*}}(t)^{m}-b_{1}(t) \phi(t)\right), \quad t \neq \tau_{k}, \\
\phi\left(\tau_{k}^{+}\right)=\left(1+h_{k}\right) \phi\left(\tau_{k}\right), \quad t=\tau_{k}, k=1,2, \ldots,
\end{gathered}
$$

where $\phi(t)$ is the solution of (34) with $\phi\left(T_{2}^{+}\right)=x\left(T_{2}^{+}\right)$. By Lemma 3 , for any $t>T_{2}$, we have $x(t) \geq \phi(t)$. From condition $\left(\mathrm{H}_{2}\right)$, we have

$$
\sum_{k=1}^{q} \ln \left(1+h_{k}\right)+\operatorname{Tm}\left[r_{1}(t)-\frac{c_{1}(t)}{k}\left(\widetilde{y_{\varepsilon}^{*}}(t)\right)^{m}\right]>0 .
$$

From inequality (35) and Lemma 4, system (34) has a unique $T$-periodic solution $x_{* \varepsilon}(t)$, which is globally stable. Since condition $\left(\mathrm{H}_{4}\right)$ holds, for the above $\varepsilon>0$ being small enough, we have $m\left[r_{1}(t)-\left(c_{1}(t) / k\right)\left(\widetilde{y_{\varepsilon}^{*}}(t)\right)^{m}\right]>0$; letting $\varepsilon \rightarrow 0$, from Lemma 6, we have $x_{* \varepsilon}(t) \rightarrow x_{*}(t)$. Letting $m_{1}=\inf \left\{x_{*}(t):\right.$ $t \in[0, T]\}$, for the above $\varepsilon$ being small enough, there exists $T_{3}>T_{2}$ such that $x(t) \geq \phi(t) \geq x_{*}(t)-\varepsilon>m_{1}-\varepsilon$, for any $t>T_{3}$. Let $\varepsilon \rightarrow 0$; we have $\lim _{t \rightarrow+\infty} \inf x(t) \geq m_{1}$. From system (3), for $t>T_{3}$ and $t \neq \tau_{k}$, we have

$$
\begin{aligned}
\dot{y}(t) \geq y(t)( & -r_{2}(t)-b_{2}(t) y(t) \\
& \left.+\frac{c_{2}(t)\left(x_{*}(t)-\varepsilon\right)}{k+\left(x_{*}(t)-\varepsilon\right)}\left(\widetilde{y_{\varepsilon}^{*}}(t)\right)^{m-1}\right) .
\end{aligned}
$$

Consider the following auxiliary system:

$$
\begin{gathered}
\dot{w}(t)=w(t)\left(-r_{2}(t)-b_{2}(t) w(t)\right. \\
\left.+\frac{c_{2}(t)\left(x_{*}(t)-\varepsilon\right)}{k+\left(x_{*}(t)-\varepsilon\right)}\left(\widetilde{y_{\varepsilon}^{*}}(t)\right)^{m-1}\right), \quad t \neq \tau_{k} \\
w\left(\tau_{k}^{+}\right)=\left(1+g_{k}\right) w\left(\tau_{k}\right), \quad t=\tau_{k}, k=1,2, \ldots,
\end{gathered}
$$

where $w(t)$ is the solution of (37) with $w\left(T_{3}^{+}\right)=y\left(T_{3}^{+}\right)$. By Lemma 3, for all $t>T_{3}$, we have $y(t) \geq w(t)$, for the above $\varepsilon>0$ being small enough. By condition $\left(\mathrm{H}_{3}\right)$, we obtain

$$
\begin{aligned}
& \sum_{k=1}^{q} \ln \left(1+g_{k}\right)+\operatorname{Tm}\left[-r_{2}+\frac{c_{2}(t)\left(x_{*}(t)-\varepsilon\right)}{k+\left(x_{*}(t)-\varepsilon\right)}\left(\widetilde{y_{\varepsilon}^{*}}(t)\right)^{m-1}\right] \\
& >0 .
\end{aligned}
$$

From inequality (38), Lemma 4, system (37) has a unique $T$-periodic solution $y_{* \varepsilon}(t)$, which is globally asymptotically stable. Since condition $\left(\mathrm{H}_{5}\right)$ holds, for the above $\varepsilon>0$ being small enough, such that $m\left[-r_{2}(t)+\left(\left(c_{2}(t)\left(x_{*}(t)-\right.\right.\right.\right.$ $\left.\left.\varepsilon)) /\left(k+\left(x_{*}(t)-\varepsilon\right)\right)\right)\left(\widetilde{y_{\varepsilon}^{*}}(t)\right)^{m-1}\right]>0$, let $\varepsilon \rightarrow 0$, and from Lemma 6, we have $y_{* \varepsilon}(t) \rightarrow y_{*}(t)$, where $y_{*}(t)$ is the unique $T$-periodic globally asymptotically stable solution of the following system:

$$
\begin{gathered}
\dot{p}(t)=p(t)\left(-r_{2}(t)-b_{2}(t) p(t)\right. \\
\left.+\frac{c_{2}(t) x_{*}(t)}{k+x_{*}(t)}\left(\widetilde{y^{*}}(t)\right)^{m-1}(t)\right), \quad t \neq \tau_{k}, \\
p\left(\tau_{k}^{+}\right)=\left(1+g_{k}\right) p\left(\tau_{k}\right), \quad t=\tau_{k}, k=1,2, \ldots
\end{gathered}
$$

Letting $m_{2}=\inf \left\{y_{*}(t): t \in[0, T]\right\}$, for the above $\varepsilon>0$ being small enough, there exists $T_{4}>T_{3}$ such that $y(t) \geq w(t)>$ $y_{*}(t)-\varepsilon$, for any $t>T_{4}$. Let $\varepsilon \rightarrow 0$; we have $\lim _{t \rightarrow+\infty} y(t) \geq$ $m_{2}$.

Theorem 12. Assume that

$$
\begin{gathered}
\sum_{k=1}^{q} \ln \left(1+g_{k}\right)+\operatorname{Tm}\left[-r_{2}(t)-\frac{c_{2}(t) x^{*}(t)}{k+x^{*}(t)}\left(y_{*}(t)\right)^{m-1}\right]<0, \\
\sum_{k=1}^{q} \ln \left(1+h_{6}\right)+\operatorname{Tm}\left[r_{1}(t)\right]>0 .
\end{gathered}
$$

Then, for any positive solution $(x(t), y(t))^{T}$ of system (3), one has

$$
\lim _{t \rightarrow+\infty} y(t)=0, \quad \lim _{t \rightarrow+\infty}\left|x(t)-x^{*}(t)\right|=0
$$

where $x^{*}(t)$ and $y_{*}(t)$ are unique T-periodic solutions of systems (9) and (39), respectively.

Proof. From system (3), for $t>T_{4}$ and $t \neq \tau_{k}$, we have

$$
\dot{y}(t) \leq y(t)\left(-r_{2}(t)-b_{2}(t) y(t)+\frac{c_{2}(t) x^{*}(t)}{k+x^{*}(t)}\left(y_{*}(t)\right)^{m-1}\right) .
$$


Consider the following auxiliary system:

$$
\begin{aligned}
& \dot{\rho}(t)=\dot{y}(t) \\
& \leq \rho(t)\left(-r_{2}(t)-b_{2}(t) \rho(t)\right. \\
& \left.\quad+\frac{c_{2}(t) x^{*}(t)}{k+x^{*}(t)}\left(\rho_{*}(t)\right)^{m-1}\right), \quad t \neq \tau_{k}, \\
& \rho\left(\tau_{k}^{+}\right)=\left(1+g_{k}\right) \rho\left(\tau_{k}\right), \quad t=\tau_{k}, \quad k=1,2, \ldots
\end{aligned}
$$

From $\left(\mathrm{H}_{6}\right)$ and Lemma 4, we have

$$
\lim _{t \rightarrow+\infty} y(t)=0
$$

Therefore, for $\varepsilon>0$ being small enough, there exists $T_{5}>0$ such that $y(t)<\varepsilon$ for any $t>T_{5}$. Since $\left(\mathrm{H}_{7}\right)$ holds, we obtain

$$
\sum_{k=1}^{q} \ln \left(1+h_{k}\right)+\operatorname{Tm}\left[r_{1}(t)-c_{1}(t) \varepsilon^{m}\right]>0 .
$$

Thus, from the first equation of system (3), we have

$$
\begin{aligned}
x(t) & \left(r_{1}(t)-b_{1}(t) x(t)-c_{1}(t) \varepsilon^{m}\right) \\
& \leq \dot{x}(t) \leq x(t)\left(r_{1}(t)-b_{1}(t) x(t)\right)
\end{aligned}
$$

for any $t>T_{5}$. Consider the following auxiliary system

$$
\begin{aligned}
\dot{\varphi}(t) & =\varphi(t)\left(r_{1}(t)-b_{1}(t) \varphi(t)-c_{1}(t) \varepsilon^{m}\right), \quad t \neq t_{k}, \\
\varphi\left(\tau_{k}^{+}\right) & =\left(1+h_{k}\right) \varphi\left(\tau_{k}\right), \quad t=\tau_{k}, \quad k=1,2, \ldots, \\
\dot{\sigma}(t) & =\sigma(t)\left(r_{1}(t)-b_{1}(t) \sigma(t)\right), \quad t \neq t_{k}, \\
\sigma\left(\tau_{k}^{+}\right) & =\left(1+h_{k}\right) \sigma\left(\tau_{k}\right), \quad t=\tau_{k}, k=1,2, \ldots
\end{aligned}
$$

From $\left(\mathrm{H}_{7}\right)$ and Lemma 4, system (46) has unique $T$-periodic solutions $\varphi_{\varepsilon}^{*}(t)$ and $\sigma^{*}(t)$, respectively, which are globally asymptotically stable. By Lemma 3, for any $\varepsilon_{1}>0$ being small enough, there exists $T_{6}>0$ such that $\varphi_{\varepsilon}^{*}(t)-\varepsilon_{1} \leq$ $x(t) \leq \sigma^{*}(t)+\varepsilon_{1}$ for any $t>T_{6}$; letting $\varepsilon \rightarrow 0$, we have $x^{*}(t)-\varepsilon_{1} \leq x(t) \leq x^{*}(t)+\varepsilon_{1}$; since $\varepsilon_{1}$ is arbitrarily small, we have $\lim _{t \rightarrow+\infty}\left|x(t)-x^{*}(t)\right|=0$.

Theorem 13. Suppose that $\left(H_{6}\right)$ holds and

$$
\sum_{k=1}^{q} \ln \left(1+h_{k}\right)+\operatorname{Tm}\left[r_{1}(t)\right]<0 .
$$

Then species $x$ and $y$ are extinct; that is,

$$
\lim _{t \rightarrow \infty} x(t)=0, \quad \lim _{t \rightarrow \infty} y(t)=0 .
$$

Proof. From $\left(\mathrm{H}_{8}\right)$ and Lemma 4, system (9) has a unique Tperiodic solution $x^{*}(t)$, and $x^{*}(t)=0$. By Lemma 3, we have $\lim _{t \rightarrow \infty} x(t) \equiv 0$; therefore, $\lim _{t \rightarrow \infty} y(t)=0$.
Theorem 14. Suppose that all the conditions of Theorem 11 hold and there exist positive constants $\alpha$ and $\delta$ such that

$$
\begin{gathered}
\min _{t \in[0, T]}\left\{\alpha b_{1}(t)-\frac{\alpha c_{1}(t)\left(M_{2}\right)^{m}}{\left.\left(k+m_{1}\right)^{2}-\frac{\delta c_{2}(t) k\left(m_{2}\right)^{m-1}}{\left(k+m_{1}\right)^{2}}\right\}>0}, \quad\left(\mathrm{H}_{9}\right)\right. \\
\min _{t \in[0, T]}\left\{\delta b_{2}(t)-\frac{\alpha c_{1}(t) m\left(m_{2}\right)^{m-1}}{k+m_{1}}\right. \\
\left.-\frac{\delta c_{2}(t) M_{1}(m-1)\left(m_{2}\right)^{m-2}}{k+m_{1}}\right\}>0 .
\end{gathered}
$$

Then system (3) has a unique positive T-periodic solution which is globally asymptotically stable.

Proof. Let $(x(t), y(t))^{T}$ and $(\bar{x}(t), \bar{y}(t))^{T}$ be any two positive solutions of system (3); for any sufficiently small $\varepsilon>0$, from Theorem 11, there is a large enough $T_{4}>0$ such that for any $t>T_{4}$, we have

$$
\begin{array}{ll}
m_{1}-\varepsilon \leq x(t), & \bar{x}(t) \leq M_{1}+\varepsilon, \\
m_{2}-\varepsilon \leq y(t), & \bar{y}(t) \leq M_{2}+\varepsilon .
\end{array}
$$

Define the Lyapunov function as follows:

$$
V(t)=\alpha|\ln x(t)-\ln \bar{x}(t)|+\delta|\ln y(t)-\ln \bar{y}(t)| .
$$

By the mean value theorem and (48), for any $t \in\left(\tau_{k}, \tau_{k+1}\right]$, $k=p, p+1, \ldots$, and $\tau_{p}>T_{4}$, we have

$$
\begin{gathered}
\frac{1}{M_{1}+\varepsilon}|x(t)-\bar{x}(t)| \leq|\ln x(t)-\ln \bar{x}(t)| \leq|x(t)-\bar{x}(t)|, \\
\frac{1}{M_{2}+\varepsilon}|y(t)-\bar{y}(t)| \leq|\ln y(t)-\ln \bar{y}(t)| \leq|y(t)-\bar{y}(t)| \\
\frac{1}{k+\bar{x}(t)}-\frac{1}{k+x(t)}=\frac{1}{\left(k+\xi_{11}(t)\right)^{2}}(\bar{x}(t)-x(t)), \\
\frac{x(t)}{k+x(t)}-\frac{k}{k+\bar{x}(t)}=\frac{k}{\left(k+\xi_{12}(t)\right)^{2}}(x(t)-\bar{x}(t)), \\
(\bar{y}(t))^{m}-(y(t))^{m}=m\left(\xi_{21}(t)\right)^{m-1}(\bar{y}(t)-y(t)) \\
(y(t))^{m-1}-(\bar{y}(t))^{m-1} \\
=(m-1)\left(\xi_{22}(t)\right)^{m-2}(y(t)-\bar{y}(t))
\end{gathered}
$$

where $\xi_{1 i}$ is suited between $x(t)$ and $\bar{x}(t), \xi_{2 i}$ is suited between $y(t)$ and $\bar{y}(t)$, and $i=1,2$. For $t>T_{4}, t \neq \tau_{k}(k=1,2, \ldots)$ 
and the above small enough $\varepsilon>0$; from conditions $\left(\mathrm{H}_{9}\right)$ and $\left(\mathrm{H}_{10}\right)$, there exist positive constants $\eta$ and $\theta$ such that

$$
\begin{gathered}
\min _{t \in[0, T]}\left\{\alpha b_{1}(t)-\frac{\alpha c_{1}(t)\left(M_{2}+\varepsilon\right)^{m}}{\left(k+m_{1}-\varepsilon\right)^{2}}\right. \\
\left.-\frac{\delta c_{2}(t) k\left(m_{2}-\varepsilon\right)^{m-1}}{\left(k+m_{1}-\varepsilon\right)^{2}}\right\}>\eta, \\
\min _{t \in[0, T]}\left\{\delta b_{2}(t)-\frac{\alpha c_{1}(t)(m)\left(m_{2}-\varepsilon\right)^{m-1}}{k+m_{1}-\varepsilon}\right. \\
\left.-\frac{\delta c_{2}(t)\left(M_{1}+\varepsilon\right)(m-1)\left(m_{2}-\varepsilon\right)^{m-2}}{k+m_{1}+\varepsilon}\right\}>\eta, \\
\eta \min \left\{\frac{m_{1}-\varepsilon}{\alpha}, \frac{m_{2}-\varepsilon}{\delta}\right\}>\theta .
\end{gathered}
$$

Calculating the upper right derivative of $V(t)$, we obtain

$$
\begin{aligned}
& D^{+} V(t)=\alpha \operatorname{sgn}(x(t)-\bar{x}(t))\left(\frac{\dot{x}(t)}{x(t)}-\frac{\dot{\bar{x}}(t)}{\overline{\bar{x}}(t)}\right) \\
& +\delta \operatorname{sgn}(y(t)-\bar{y}(t))\left(\frac{\dot{y}(t)}{y_{1}(t)}-\frac{\dot{\bar{y}}(t)}{\bar{y}(t)}\right) \\
& =\alpha \operatorname{sgn}(x(t)-\bar{x}(t)) \\
& \times\left[-b_{1}(t)(x(t)-\bar{x}(t))\right. \\
& \left.-c_{1}(t)\left(\frac{(y(t))^{m}}{k+x(t)}-\frac{(\bar{y}(t))^{m}}{k+\bar{x}(t)}\right)\right] \\
& +\delta \operatorname{sgn}(y(t)-\bar{y}(t)) \\
& \times\left[-b_{2}(t)(y(t)-\bar{y}(t))\right. \\
& -c_{2}(t)\left(\frac{x(t)(y(t))^{m-1}}{k+x(t)}\right. \\
& \left.\left.-\frac{\bar{x}(t)(\bar{y}(t))^{m-1}}{k+\bar{x}(t)}\right)\right] \\
& =-\alpha b_{1}(t)|x(t)-\bar{x}(t)|-\delta b_{2}(t)|y(t)-\bar{y}(t)| \\
& +\alpha c_{1}(t) \operatorname{sgn}(x(t)-\bar{x}(t)) \\
& \times\left(\frac{(\bar{y}(t))^{m}}{k+\bar{x}(t)}-\frac{(y(t))^{m}}{k+x(t)}\right) \\
& +\delta c_{2}(t) \operatorname{sgn}(y(t)-\bar{y}(t)) \\
& \times\left(\frac{x(t)(y(t))^{m-1}}{k+x(t)}-\frac{\bar{x}(t)(\bar{y}(t))^{m-1}}{k+\bar{x}(t)}\right) .
\end{aligned}
$$

In view of

$$
\begin{aligned}
c_{1}(t) \operatorname{sgn}(x(t)-\bar{x}(t))\left(\frac{(\bar{y}(t))^{m}}{k+\bar{x}(t)}-\frac{(y(t))^{m}}{k+x(t)}\right) \\
=c_{1}(t) \operatorname{sgn}(x(t)-\bar{x}(t)) \\
\times\left[\frac{(\bar{y}(t))^{m}}{k+\bar{x}(t)}-\frac{(\bar{y}(t))^{m}}{k+x(t)}+\frac{(\bar{y}(t))^{m}}{k+x(t)}+\frac{(y(t))^{m}}{k+x(t)}\right] \\
=c_{1}(t) \operatorname{sgn}(x(t)-\bar{x}(t))(\bar{y}(t))^{m} \\
\times\left[\frac{1}{\left(k+\xi_{11}\right)^{2}}(\bar{x}(t)-x(t))\right. \\
\left.+\frac{m\left(\xi_{21}(t)\right)^{m-1}}{k+x(t)}(\bar{y}(t)-y(t))\right],
\end{aligned}
$$

$$
\begin{aligned}
\mathcal{c}_{2}(t) \operatorname{sgn}(y(t)-\bar{y}(t))\left(\frac{x(t)(y(t))^{m-1}}{k+x(t)}-\frac{\bar{x}(t)(\bar{y}(t))^{m-1}}{k+\bar{x}(t)}\right) \\
=c_{2}(t) \operatorname{sgn}(y(t)-\bar{y}(t)) \\
\times\left[\frac{x(t)(y(t))^{m-1}}{k+x_{1}(t)}-\frac{x(t)(\bar{y}(t))^{m-1}}{k+x(t)}\right. \\
\left.\quad+\frac{x(t)(\bar{y}(t))^{m-1}}{k+x(t)}-\frac{\bar{x}(t)(\bar{y}(t))^{m-1}}{k+\bar{x}(t)}\right] \\
=c_{2}(t) \operatorname{sgn}(y(t)-\bar{y}(t)) \\
\times\left[\frac{x(t)(m-1)\left(\xi_{22}(t)\right)^{m-2}}{k+x(t)}(y(t)-\bar{y}(t))\right. \\
\left.\quad+\frac{k}{\left(k+\xi_{12}(t)\right)^{2}}(\bar{y}(t))^{m-1}(x(t)-\bar{x}(t))\right],
\end{aligned}
$$

substituting (53) and (54) into (52), we have

$D^{+} V(t)$

$$
\begin{gathered}
\leq-\left(\alpha b_{1}(t)-\alpha c_{1}(t) \frac{(\bar{y}(t))^{m}}{\left(k+\xi_{11}\right)^{2}(t)}\right. \\
\left.-\frac{\delta c_{2}(t) k(\bar{y}(t))^{m-1}}{\left(k+\xi_{12}^{2}\right)}\right) \\
\times|x(t)-\bar{x}(t)| \\
-\left[\delta b_{2}(t)-\frac{\alpha c_{1}(t)(\bar{y}(t))^{m} m\left(\xi_{21}^{m-1}(t)\right)}{k+x}\right] \\
\left.-\frac{\delta c_{2}(t) x(t)(m-1)\left(\xi_{22}\right)^{m-2}(t)}{k+x}\right]
\end{gathered}
$$


TABLE 2: The effects of $h_{k}$ and $g_{k}$ on the dynamical behavior of system (3).

\begin{tabular}{lccccc}
\hline Case & $h_{k}$ & $g_{k}$ & $x$ & $y$ & Figure \\
\hline 1 & 0.2 & 0.03 & Permanence & Permanence & Figure 1 \\
2 & -0.2 & -0.6 & Permanence & Extinction & Figure 2 \\
\hline
\end{tabular}

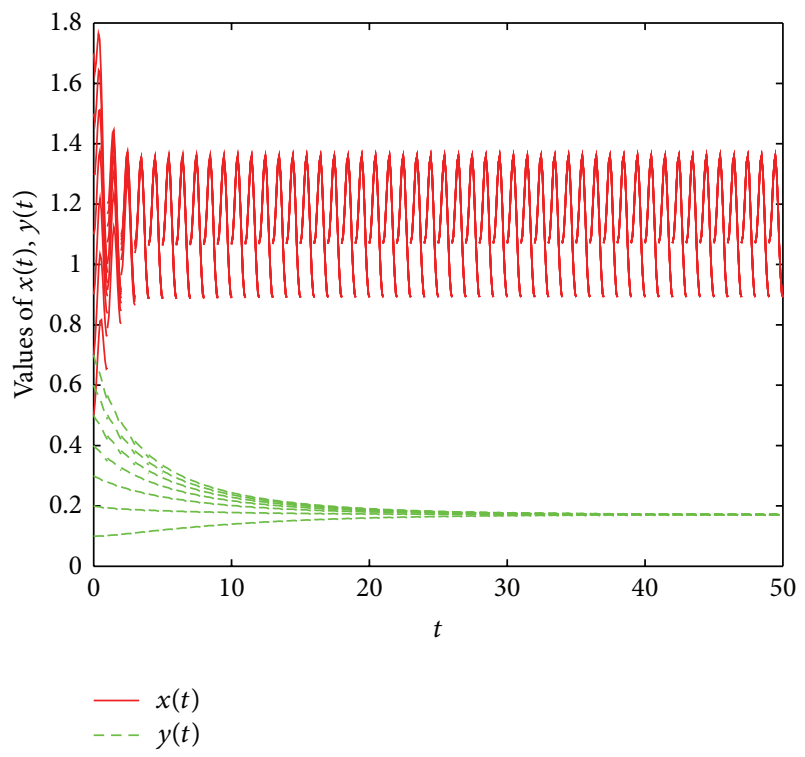

Figure 1: Time series of $x(t)$ and $y(t)$.

$$
\begin{aligned}
& \times|y(t)-\bar{y}(t)| \\
& \leq-\left[\alpha b_{1}(t)-\alpha c_{1}(t) \frac{\left(M_{2}+\varepsilon\right)^{m}}{\left(k+m_{1}-\varepsilon\right)^{2}}\right. \\
& \left.-\frac{\delta c_{2}(t) k\left(m_{2}-\varepsilon\right)^{m-1}}{\left(k+m_{1}-\varepsilon\right)^{2}}\right]|x(t)-\bar{x}(t)| \\
& -\left[\delta b_{2}(t)-\frac{\alpha c_{1}(t)\left(M_{2}+\varepsilon\right)^{m} m\left(m_{2}-\varepsilon\right)^{m-1}}{k+m_{1}-\varepsilon}\right. \\
& \left.-\frac{\delta c_{2}(t)\left(M_{1}+\varepsilon\right)(m-1)\left(m_{2}-\varepsilon\right)^{m-2}}{k+m_{1}-\varepsilon}\right] \\
& \times|y(t)-\bar{y}(t)| \\
& \leq-\eta\left(\frac{m_{1}-\varepsilon}{\alpha} \alpha|\ln x(t)-\ln \bar{x}(t)|\right. \\
& \left.+\frac{m_{2}-\varepsilon}{\delta} \delta|\ln y(t)-\ln \bar{y}(t)|\right) .
\end{aligned}
$$

For all $t \in R_{+}$and $t=\tau_{k}^{+}(k=1,2, \ldots)$, we have

$$
\begin{aligned}
\dot{V}\left(\tau_{k}^{+}\right) & =\alpha\left|\ln x\left(\tau_{k}^{+}\right)-\ln \bar{x}\left(\tau_{k}^{+}\right)\right|+\delta\left|\ln y\left(\tau_{k}^{+}\right)-\ln \bar{y}\left(\tau_{k}^{+}\right)\right| \\
& =\alpha\left|\ln \left(1+h_{k}\right) x\left(\tau_{k}\right)-\ln \left(1+h_{k}\right) \bar{x}\left(\tau_{k}\right)\right|
\end{aligned}
$$

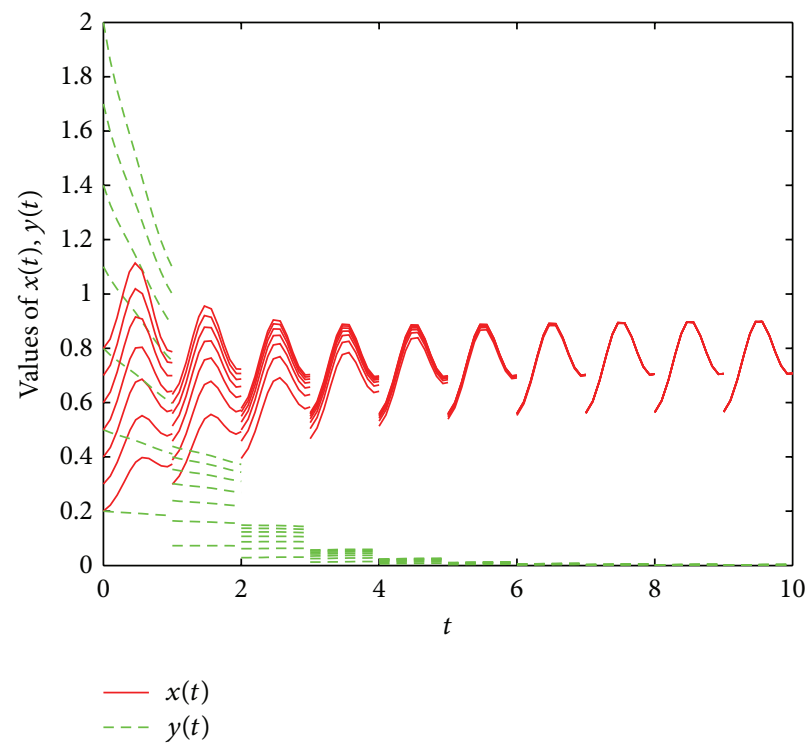

Figure 2: Time series of $x(t)$ and $y(t)$.

TABLE 3: The effects of $h_{k}$ on the dynamical behavior of system (3).

\begin{tabular}{lclc}
\hline Case & $h_{k}$ & $g_{k}$ & Figure \\
\hline- & Bifurcation parameter & 0.3 & Figure 3(a) \\
1 & -0.4 & 0.3 & Figure 3(b) \\
2 & 0 & 0.3 & Figure 3(c) \\
3 & 0.6 & 0.3 & Figure 3(d) \\
\hline & \\
& $+\delta\left|\ln \left(1+g_{k}\right) y\left(\tau_{k}\right)-\ln \left(1+g_{k}\right) \bar{y}\left(\tau_{k}\right)\right|$ \\
& $=V\left(\tau_{k}\right)$.
\end{tabular}

Hence, $V(t)$ is continuous for all $t>\tau_{p}>T_{4}$. The above analysis shows that, for all $t>\tau_{p}>T_{4}$,

$$
D^{+} V(t) \leq-\theta V(t)
$$

and we further have

$$
V(t) \leq V\left(\tau_{p}\right) \exp \left(-\theta\left(t-\tau_{p}\right)\right) .
$$

It is obvious that $V(t) \rightarrow 0$ as $t \rightarrow+\infty$; that is,

$$
\begin{aligned}
& \lim _{t \rightarrow+\infty}|\ln x(t)-\ln \bar{x}(t)|=0, \\
& \lim _{t \rightarrow+\infty}|\ln y(t)-\ln \bar{y}(t)|=0 .
\end{aligned}
$$

We further have

$$
\lim _{t \rightarrow+\infty}|x(t)-\bar{x}(t)|=0, \quad \lim _{t \rightarrow+\infty}|y(t)-\bar{y}(t)|=0 .
$$




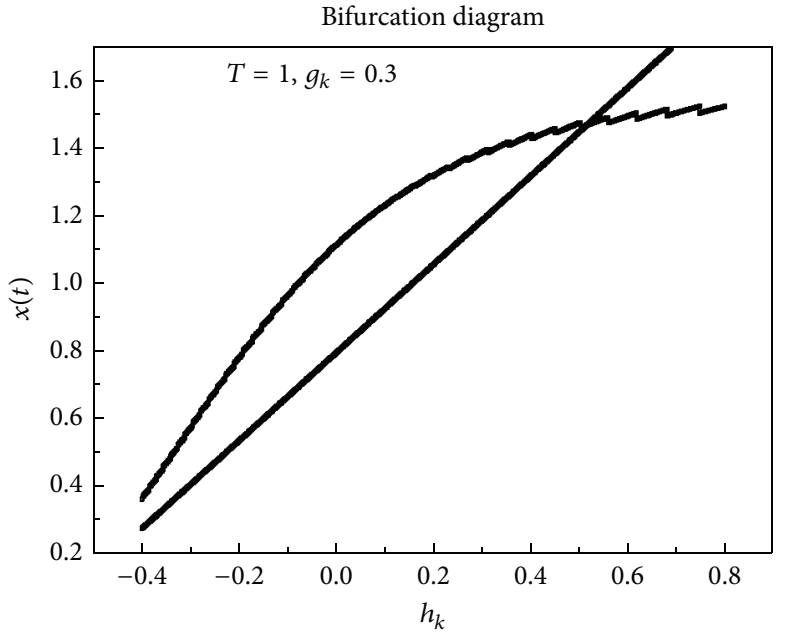

(a)

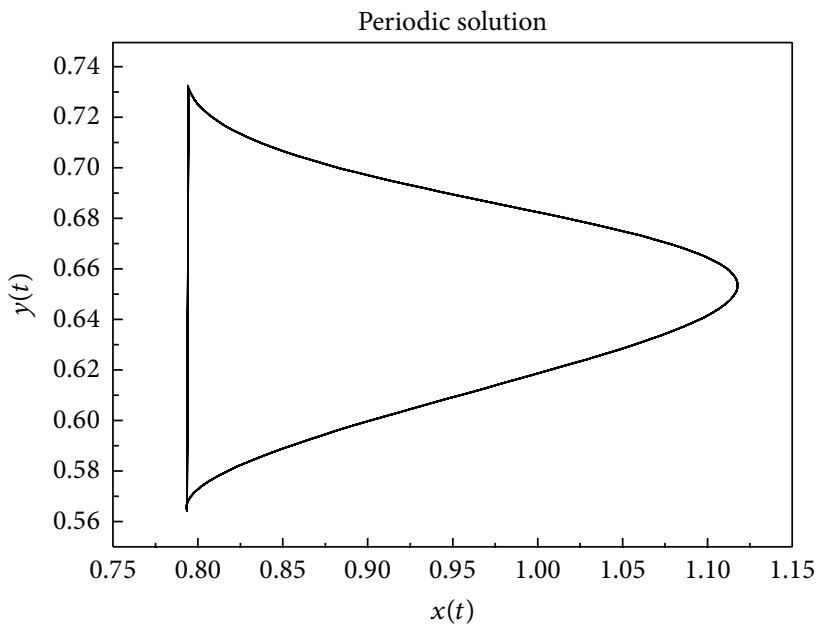

(c)

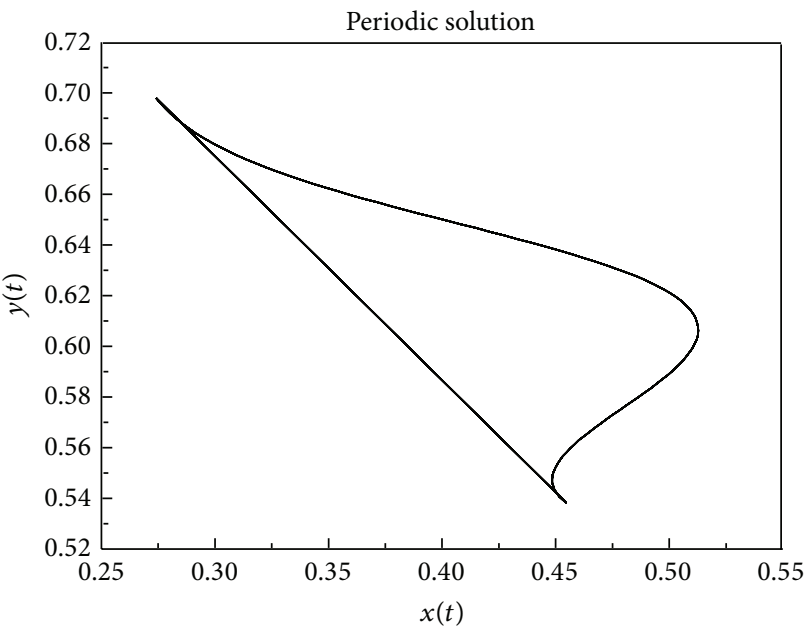

(b)

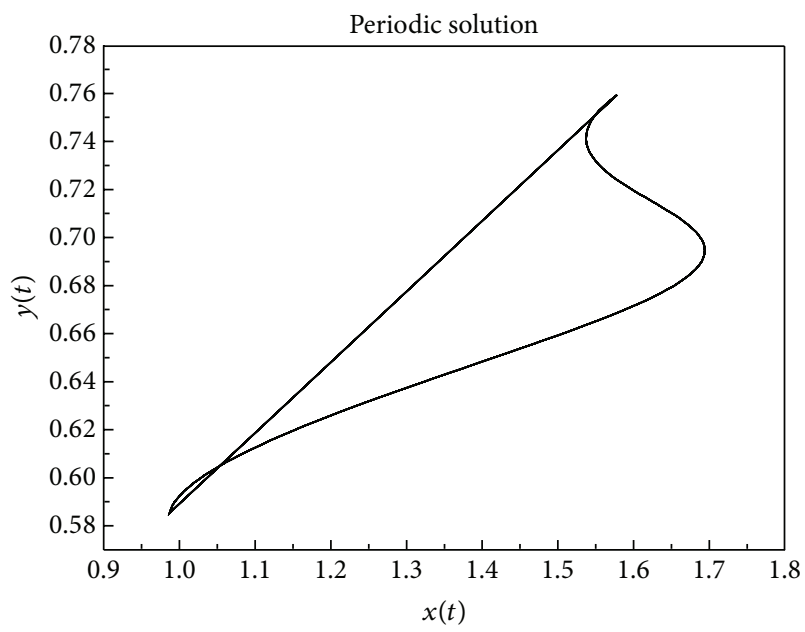

(d)

FIGURE 3: (a) Bifurcation diagram of $h_{k}$ when $g_{k}$ is fixed at 0.3 ; (b)-(d) phase portraits.

Choose constants $A=\min \left\{m_{1}, m_{2}\right\}$ and $B=\max \left\{M_{1}, M_{2}\right\}$. By Theorem 11, we have

$$
A \leq x(t), y(t), \bar{x}(t), \bar{y}(t) \leq B, \quad \forall t \geq T_{4} .
$$

Now let us consider the $\left(\bar{x}\left(m T, z_{0}\right), \bar{y}\left(m T, z_{0}\right)\right)=z\left(m T, z_{0}\right)$, $m=1,2, \ldots$, and $z_{0}=(\bar{x}(0), \bar{y}(0))$. It is compact in the domain $[A, B]^{2}$, since $A \leq \bar{x}(t)$ and $\bar{y}(t) \leq B$ for all $t \geq T_{4}$. Let $\bar{z}$ be a limit point of this sequence $\bar{z}=\lim _{n \rightarrow \infty} z\left(m_{n} T, z_{0}\right)$; then we have $z(T, \bar{z})=\bar{z}$. In fact, since $z\left(T, z\left(m_{n} T, z_{0}\right)\right)=$ $z\left(m_{n} T, z\left(T, z_{0}\right)\right)$ and $z\left(m_{n} T, z\left(T, z_{0}\right)\right)-z\left(m_{n} T, z_{0}\right) \rightarrow 0$ as $m_{n} \rightarrow \infty$, we have

$$
\begin{aligned}
\| z(T, \bar{z}) & -\bar{z} \|_{[A, B]^{2}} \\
\leq & \left\|z(T, \bar{z})-z\left(T, z\left(m_{n} T, z_{0}\right)\right)\right\|_{[A, B]^{2}} \\
& +\left\|z\left(T, z\left(m_{n} T, z_{0}\right)\right)-z\left(m_{n} T, z_{0}\right)\right\|_{[A, B]^{2}} \\
& +\left\|z\left(m_{n} T, z_{0}\right)-\bar{z}\right\|_{[A, B]^{2}} \longrightarrow 0 .
\end{aligned}
$$

The sequence $z\left(m T, z_{0}\right)(m=1,2, \ldots)$ has a unique limit point; otherwise, let the sequence have two limit points $\bar{z}=$ $\lim _{n \rightarrow \infty} z\left(m_{n} T, z_{0}\right)$ and $\widetilde{z}=\lim _{n \rightarrow \infty} z\left(m_{n} T, z_{0}\right)$. Then, taking into account (60) and $\widetilde{z}=z\left(m_{n} T, \widetilde{z}\right)$, we have

$$
\begin{aligned}
\|\bar{z}-\tilde{z}\|_{[A, B]^{2}} \leq & \left\|\bar{z}-Z\left(m_{n} T, z_{0}\right)\right\|_{[A, B]^{2}} \\
+\| Z & \left(m_{n} T, z_{0}\right)-\widetilde{z} \|_{[A, B]^{2}} \\
& \longrightarrow 0, \quad n \longrightarrow \infty,
\end{aligned}
$$

and hence $\bar{z}=\widetilde{z}$; the solution $\left(\bar{x}\left(t, x_{0}\right), \bar{y}\left(t, y_{0}\right)\right)$ is the unique periodic solution of system (3). By (60), it is globally asymptotically stable. This completes the proof of Theorem 14 .

\section{Numerical Simulations}

In system (3), we take $T=1, q=1$, which means that system (3) is a one-period system with one impulse perturbation during one period. 


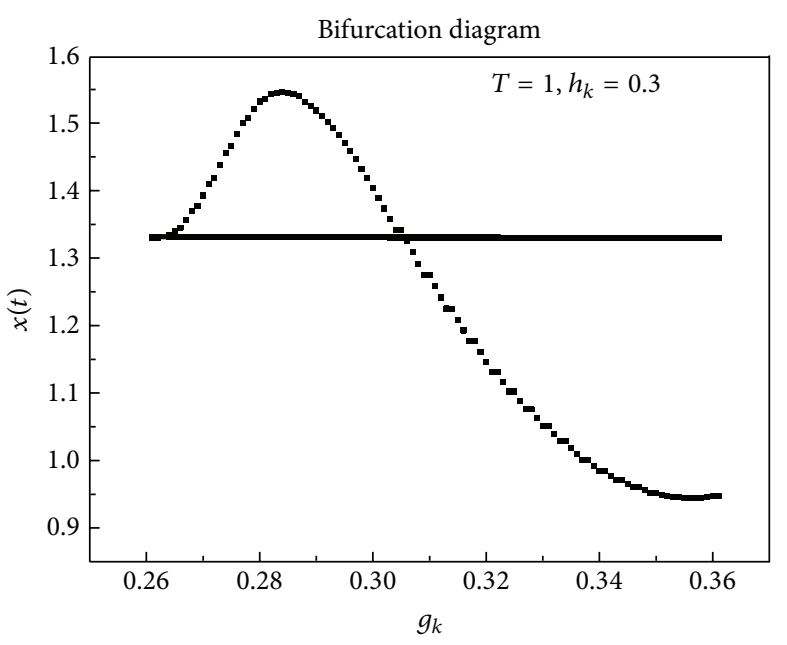

(a)

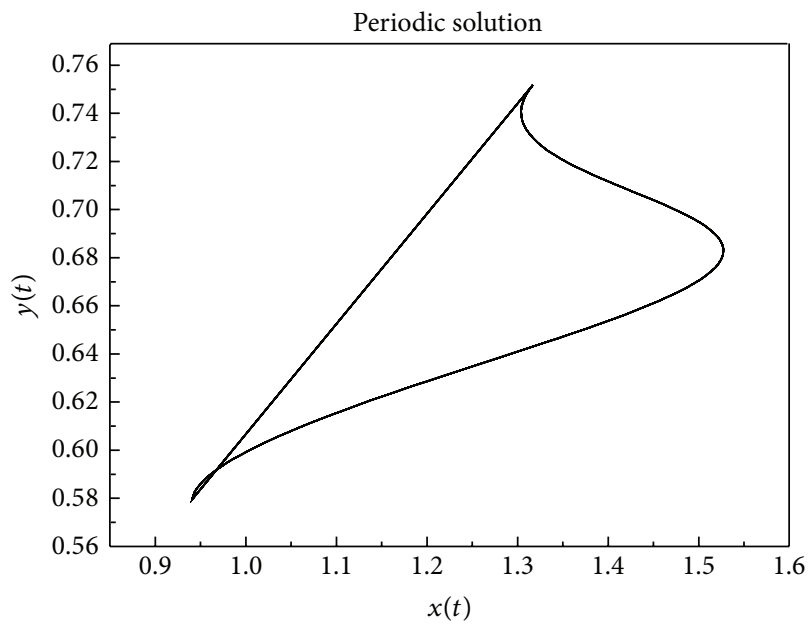

(c)

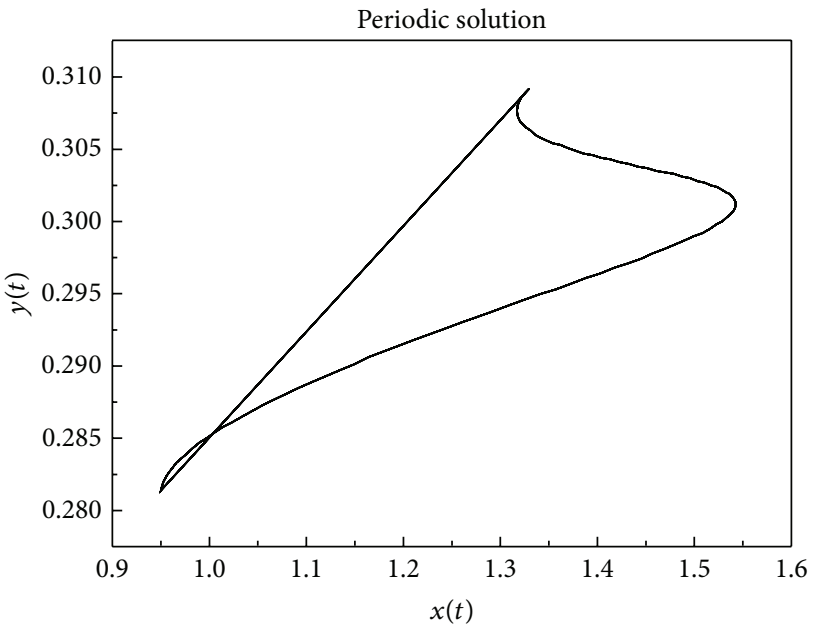

(b)

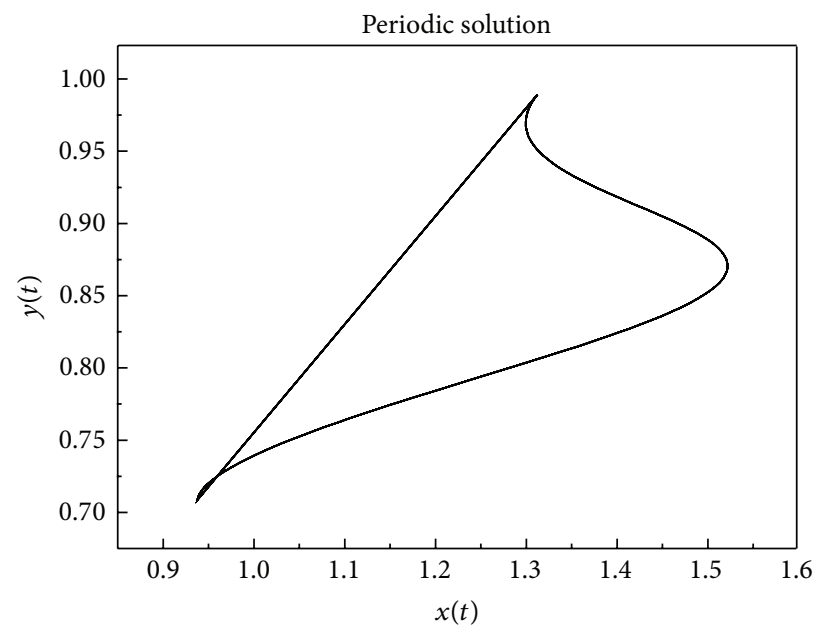

(d)

FIgURE 4: (a) Bifurcation of $g_{k}$ when $h_{k}$ is fixed at 0.4; (b)-(d) phase portraits.

TABLE 4: The effects of $g_{k}$ on the dynamical behavior of system (3).

\begin{tabular}{llcc}
\hline Case & $h_{k}$ & $g_{k}$ & Figure \\
\hline- & 0.4 & Bifurcation parameter & Figure 4(a) \\
1 & 0.4 & 0.1 & Figure 4(b) \\
2 & 0.4 & 0.3 & Figure 4(c) \\
3 & 0.4 & 0.4 & Figure 4(d) \\
\hline
\end{tabular}

In case $1,\left(\mathrm{H}_{1}\right)-\left(\mathrm{H}_{5}\right)$ are, respectively, equivalent to the following inequalities:

$$
\begin{gathered}
-\sum_{k=1}^{q} \ln \left(1+g_{k}\right)+\operatorname{Tm}\left[r_{2}(t)\right]=0.0004>0 \\
\sum_{k=1}^{q} \ln \left(1+h_{k}\right)+\operatorname{Tm}\left[r_{1}(t)-c_{1}(t)\left(\widetilde{y^{*}}(t)\right)^{m}\right] \\
>1.143>0
\end{gathered}
$$

$$
\begin{aligned}
\sum_{k=1}^{q} \ln \left(1+g_{k}\right)+\operatorname{Tm}\left[-r_{2}(t)+\frac{c_{2}(t) x_{*}(t)}{k+x_{*}(t)} \widetilde{y^{*}}(t)^{m-1}\right] \\
>0.0536>0 . \\
m\left[r_{1}(t)-c_{1}(t)\left(\widetilde{y^{*}}(t)\right)^{m}\right]>0.961>0, \\
m\left[-r_{2}(t)+\frac{c_{2}(t) x_{*}(t)}{k+x_{*}(t)} \widetilde{y^{*}}(t)^{m-1}\right]>0.024>0 .
\end{aligned}
$$

Taking $\alpha=1.5, \delta=1$, conditions $\left(\mathrm{H}_{9}\right)$ and $\left(\mathrm{H}_{10}\right)$ are, respectively, equivalent to the following inequalities:

$$
\begin{aligned}
& \min _{t \in[0, T]}\left\{\alpha b_{1}(t)-\frac{\alpha c_{1}(t)\left(M_{2}\right)^{m}}{\left(k+m_{1}\right)^{2}}-\frac{\delta c_{2}(t) k\left(m_{2}\right)^{m-1}}{\left(k+m_{1}\right)^{2}}\right\} \\
& \simeq 1.221>0, \\
& \min _{t \in[0, T]}\left\{\delta b_{2}(t)-\frac{\alpha c_{1}(t)(m)\left(m_{2}\right)^{m-1}}{k+m_{1}}\right.
\end{aligned}
$$


TABLE 5: The effects of impulse period $T$ on the dynamical behavior of system (3).

\begin{tabular}{lcccc}
\hline Case & $T$ & $x$ & $y$ & Figure \\
\hline 1 & 1 & Periodic & Periodic & Figures 5(a) and 5(b) \\
2 & 1.002 & Almost periodic & Almost periodic & Figure 5(c) \\
3 & 1.014 & Chaotic & Chaotic & Figure 5(d) \\
\hline
\end{tabular}

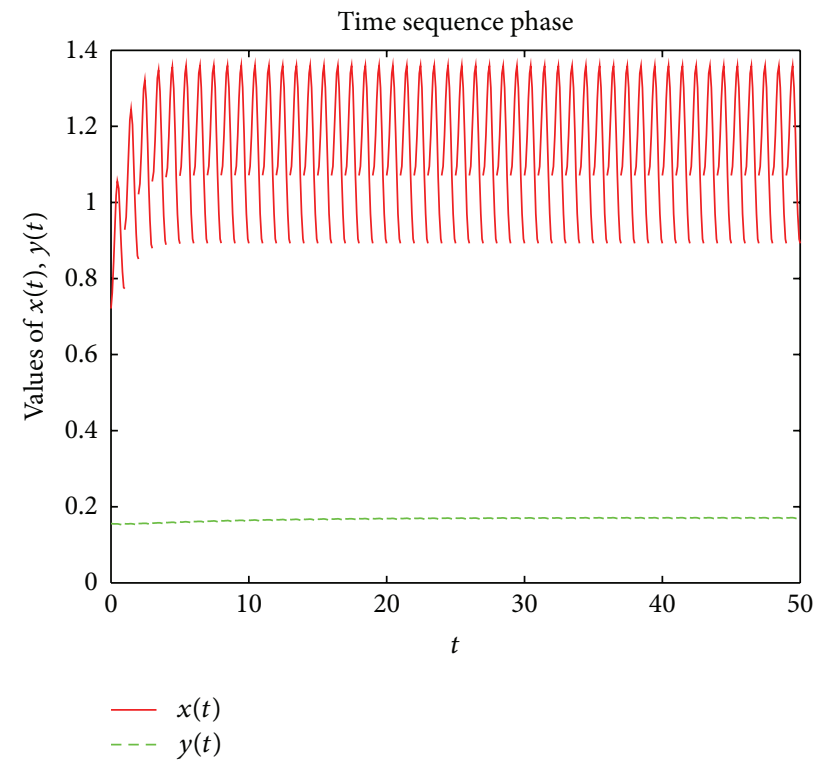

(a)

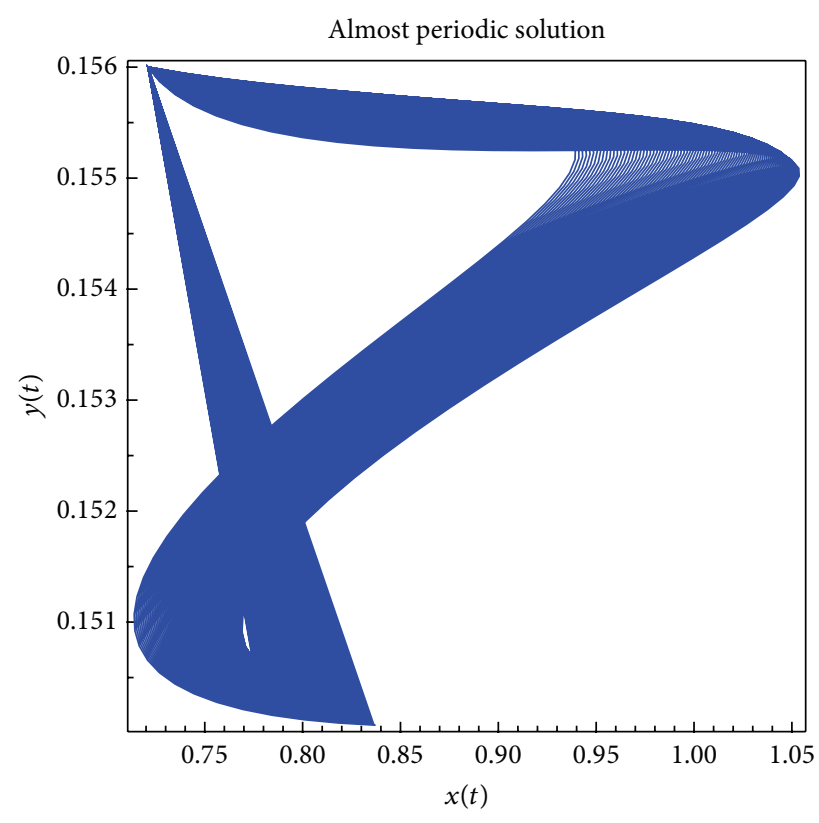

(c)

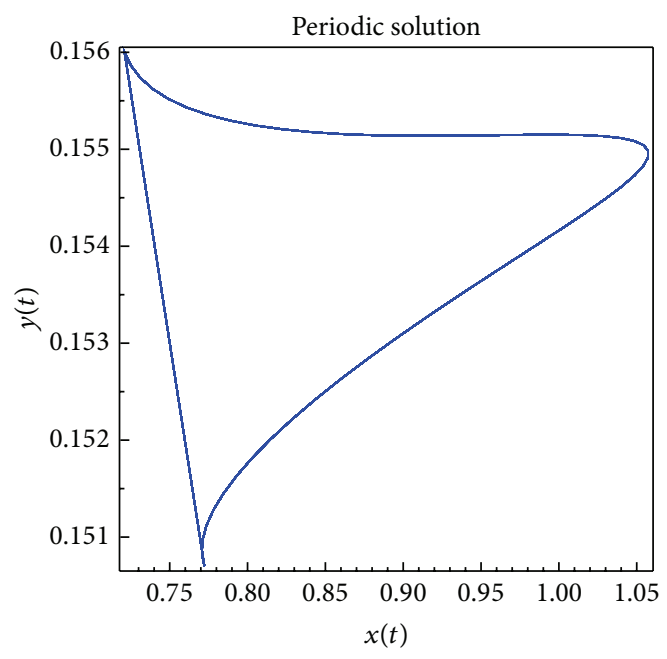

(b)

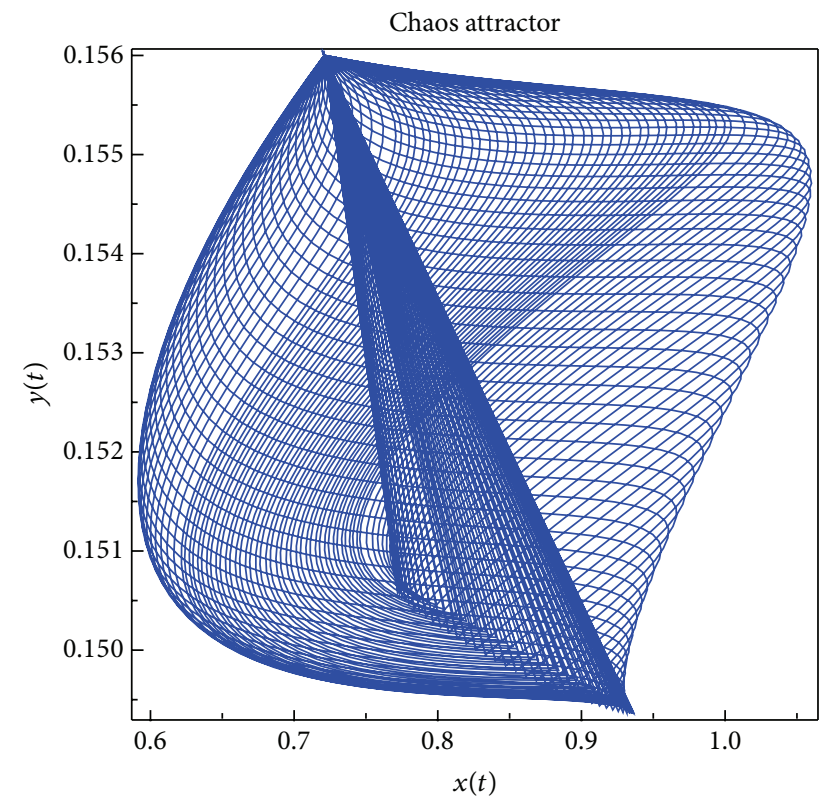

(d)

FIGURE 5: Dynamical behavior of system (3); here, we take initial value $(x(0), y(0))=(0.721,0.156)$.

$$
\left.-\frac{\delta c_{2}(t) M_{1}(m-1)\left(m_{2}\right)^{m-2}}{k+m_{1}}\right\}
$$$$
\simeq 0.5498>0 .
$$

By calculation, conditions $\left(\mathrm{H}_{1}\right)-\left(\mathrm{H}_{5}\right),\left(\mathrm{H}_{9}\right)$, and $\left(\mathrm{H}_{10}\right)$ hold; from Theorem 11 and Theorem 14, system (3) is globally asymptotically stable. Numerical simulations of these results can be seen in Figure 1.

Figure 1 shows the dynamical behavior of the solutions $(x(t), y(t))$ with seven group initial values $(x(0), y(0))=$ 
$(0.5,0.1),(0.6,0.2),(0.7,0.3),(0.8,0.4),(0.9,0.5),(1.0,0.6)$, and $(1.1,0.7)$, respectively. From Figure 1 , we can obtain that there exists a unique positive periodic solution of system (3) such that any solution of system (3) with initial value $(x(0), y(0))$ tends to the positive periodic solution as $t \rightarrow \infty$.

In case 2 , conditions $\left(\mathrm{H}_{6}\right)$ and $\left(\mathrm{H}_{7}\right)$ are, respectively, equivalent to the following inequalities:

$$
\begin{aligned}
\sum_{k=1}^{q} \ln ( & \left.1+g_{k}\right)+\operatorname{Tm}\left[-r_{2}(t)-\frac{c_{2}(t) x^{*}(t)}{k+x^{*}(t)}\left(y_{*}(t)\right)^{m-1}\right] \\
= & -0.9463<0, \\
& \sum_{k=1}^{q} \ln \left(1+h_{k}\right)+\operatorname{Tm}\left[r_{1}(t)\right]=0.7769>0 .
\end{aligned}
$$

By calculation, conditions $\left(\mathrm{H}_{6}\right)$ and $\left(\mathrm{H}_{7}\right)$ hold. From Theorem 12, we have that species $y$ will be extinct and species $x$ will be globally asymptotically stable. Numerical simulations of these results are plotted in Figure 2.

Figure 2 shows the dynamical behavior of the solutions $(x(t), y(t))$ with seven group initial values $(x(0), y(0))=$ $(0.2,0.2),(0.3,0.5),(0.4,0.8),(0.5,1.1),(0.6,1.4),(0.7,1.7)$, and $(0.8,2.0)$, respectively. From Figure 2 , we can see that species $y$ will be extinct and species $x$ will be globally asymptotically stable (Table 2 ).

Furthermore, we investigate the effects of impulse coefficients $h_{k}$ and $g_{k}$ on the dynamical behavior of system (3); we keep the parameter $g_{k}$ fixed at $g_{k}=0.3$. From Table 3 , it is found that the impulse coefficient $h_{k}$ has an important influence on the sizes of prey and predator populations. Therefore, we plot the bifurcation diagram of system (3) when $h_{k} \in[-0.4,0.8]$; see Figure $3(\mathrm{a})$; it is very clear that the number of prey populations increases with increasing parameter $h_{k}$.

On the other hand, we keep the parameter $h_{k}$ fixed at $h_{k}=0.4$. For $0.26 \leq g_{k} \leq 0.36$, the effects of $g_{k}$ on the dynamical behavior of system (3) have been also investigated. From Table 4 , it is found that the impulse coefficient $g_{k}$ has an important influence on the sizes of prey and predator populations.

Moreover, on increasing the period of impulse perturbations, some interesting phenomena happen; the details are given in Table 5.

\section{Conclusion}

In this paper, a class of Holling type II predator-prey systems with mutual interference and impulses is studied. Some sufficient conditions for the permanence and extinction of the system and existence and uniqueness of positive periodic solution are obtained. These obtained results are new and the complement to previously known results. From the view point of biology, the mathematical results are full of biological meanings and can be used to provide reliable foundation for making control strategy. The conditions of Theorem 13 show that human activities may save the extinct species living in a week environment.
From the numerical simulations, we find that the behavior of impulse perturbations of species has an important influence on the system. Besides, the dynamics of species are very sensitive with the period matching between species' intrinsic disciplinarians and the perturbations from the variable environment. If the periods between individual growth and impulse perturbations match well, the dynamics behavior of species periodically changes. If they mismatch each other, the dynamic behaviors would change from period to period until there is chaos. It is very consistent with real ecosystems, which helps us interpret the diversity and many phenomena of ecosystems.

\section{Conflict of Interests}

The authors declare that there is no conflict of interests regarding the publication of this paper.

\section{Acknowledgments}

This work was supported by the National Natural Science Foundation of China (Grant nos. 11361059, 10901130, 11271312, and 11371287), the Natural Science Foundation of Xinjiang (Grant no. 2012211B07), and the Scientific Research Programmes of Colleges in Xinjiang (XJEDU2013I03).

\section{References}

[1] A. A. Berryman, "The origins and evolution of predator-prey theory," Ecology, vol. 73, no. 5, pp. 1530-1535, 1992.

[2] F.-d. Chen and J.-1. Shi, "Periodicity in a nonlinear predatorprey system with state dependent delays," Acta Mathematicae Applicatae Sinica, vol. 21, no. 1, pp. 49-60, 2005.

[3] C. Shen, "Permanence and global attractivity of the foodchain system with Holling IV type functional response," Applied Mathematics and Computation, vol. 194, no. 1, pp. 179-185, 2007.

[4] L. Zhang and Z. Teng, "Permanence for a delayed periodic predator-prey model with prey dispersal in multi-patches and predator density-independent," Journal of Mathematical Analysis and Applications, vol. 338, no. 1, pp. 175-193, 2008.

[5] F. Chen and X. Cao, "Existence of almost periodic solution in a ratio-dependent Leslie system with feedback controls," Journal of Mathematical Analysis and Applications, vol. 341, no. 2, pp. 1399-1412, 2008.

[6] X. Song and Y. Li, "Dynamic behaviors of the periodic predator-prey model with modified Leslie-Gower Holling-type II schemes and impulsive effect," Nonlinear Analysis. Real World Applications. An International Multidisciplinary Journal, vol. 9, no. 1, pp. 64-79, 2008.

[7] X. Zhou, X. Shi, and X. Song, "Analysis of nonautonomous predator-prey model with nonlinear diffusion and time delay," Applied Mathematics and Computation, vol. 196, no. 1, pp. 129136, 2008.

[8] X. Meng, W. Xu, and L. Chen, "Profitless delays for a nonautonomous Lotka-Volterra predator-prey almost periodic system with dispersion," Applied Mathematics and Computation, vol. 188, no. 1, pp. 365-378, 2007.

[9] S. Liu and J. Zhang, "Coexistence and stability of predatorprey model with Beddington-DeAngelis functional response 
and stage structure," Journal of Mathematical Analysis and Applications, vol. 342, no. 1, pp. 446-460, 2008.

[10] X. Ding and J. Jiang, "Multiple periodic solutions in delayed Gause-type ratio-dependent predator-prey systems with nonmonotonic numerical responses," Mathematical and Computer Modelling, vol. 47, no. 11-12, pp. 1323-1331, 2008.

[11] F. Han and Q. Wang, "Existence of multiple positive periodic solutions for differential equation with state-dependent delays," Journal of Mathematical Analysis and Applications, vol. 324, no. 2, pp. 908-920, 2006.

[12] C. Feng and P. Wang, "The existence of almost periodic solutions of some delay differential equations," Computers \& Mathematics with Applications. An International Journal, vol. 47, no. 8-9, pp. 1225-1231, 2004.

[13] X. Yang and R. Yuan, "Global attractivity and positive almost periodic solution for delay logistic differential equation," Nonlinear Analysis: Theory, Methods \& Applications, vol. 68, no. 1, pp. 54-72, 2008.

[14] X. Yang, "Global attractivity and positive almost periodic solution of a single species population model," Journal of Mathematical Analysis and Applications, vol. 336, no. 1, pp. 111126, 2007.

[15] M. P. Hassel, "Density dependence in single-species population," Journal of Animal Ecology, vol. 44, pp. 283-295, 1975.

[16] L. S. Chen, Mathematics Ecology Model and Reserch Methods, Science Press, Beijing, China, 1998, (Chinese).

[17] K. Wang, "Existence and global asymptotic stability of positive periodic solution for a predator-prey system with mutual interference," Nonlinear Analysis: Real World Applications, vol. 10, no. 5, pp. 2774-2783, 2009.

[18] K. Wang, "Permanence and global asymptotical stability of a predator-prey model with mutual interference," Nonlinear Analysis: Real World Applications, vol. 12, no. 2, pp. 1062-1071, 2011.

[19] L. Chen, F. Chen, and Y. Wang, "Influence of predator mutual interference and prey refuge on Lotka-Volterra predator-prey dynamics," Communications in Nonlinear Science and Numerical Simulation, vol. 18, no. 11, pp. 3174-3180, 2013.

[20] Z. Ma, F. Chen, C. Wu, and W. Chen, "Dynamic behaviors of a Lotka-Volterra predator-prey model incorporating a prey refuge and predator mutual interference," Applied Mathematics and Computation, vol. 219, no. 15, pp. 7945-7953, 2013.

[21] C. S. Holling, "The functional response of predator to prey density and its role in mimicry and population regulation," Memoirs of the Entomological Society of Canada, vol. 97, no. 45, pp. 1-60, 1965.

[22] K. Wang and Y. Zhu, "Global attractivity of positive periodic solution for a Volterra model," Applied Mathematics and Computation, vol. 203, no. 2, pp. 493-501, 2008.

[23] L. Zhang, Z. Teng, and Z. Liu, "Survival analysis for a periodic predatory-prey model with prey impulsively unilateral diffusion in two patches," Applied Mathematical Modelling, vol. 35, no. 9, pp. 4243-4256, 2011.

[24] S. H. Saker and J. O. Alzabut, "Existence of periodic solutions, global attractivity and oscillation of impulsive delay population model," Nonlinear Analysis: Real World Applications, vol. 8, no. 4, pp. 1029-1039, 2007.

[25] Z. Li, F. Chen, and M. He, "Permanence and global attractivity of a periodic predator-prey system with mutual interference and impulses," Communications in Nonlinear Science and Numerical Simulation, vol. 17, no. 1, pp. 444-453, 2012.
[26] L. Wang, Z. Liu, Jinghui, and L. Chen, "Impulsive diffusion in single species model," Chaos, Solitons and Fractals, vol. 33, no. 4, pp. 1213-1219, 2007.

[27] G. Peng, Y. Jiang, and C. Li, "Bifurcations of a Holling-type II predator-prey system with constant rate harvesting," International Journal of Bifurcation and Chaos in Applied Sciences and Engineering, vol. 19, no. 8, pp. 2499-2514, 2009.

[28] B. Kang, B. Liu, and L. Xu, "Dynamics of an inshore-offshore fishery model with impulsive pollutant input in inshore area," Nonlinear Dynamics, vol. 67, no. 4, pp. 2353-2362, 2012.

[29] V. Laksbmikanham, D. D. Bainov, and P. S. Simeonov, Theory of Impulsive Differential Equations, World Science, Singapore, 1989.

[30] F. Zhang, S. Gao, and Y. Zhang, "Effects of pulse culling on population growth of migratory birds and economical birds," Nonlinear Dynamics, vol. 67, no. 1, pp. 767-779, 2012.

[31] M. He, Z. Li, and F. Chen, "Permanence, extinction and global attractivity of the periodic Gilpin-Ayala competition system with impulses," Nonlinear Analysis: Real World Applications, vol. 11, no. 3, pp. 1537-1551, 2010. 


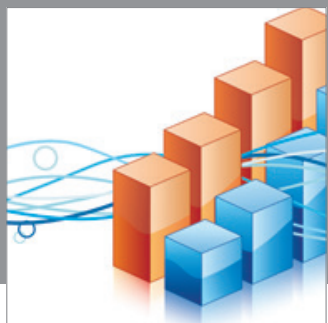

Advances in

Operations Research

mansans

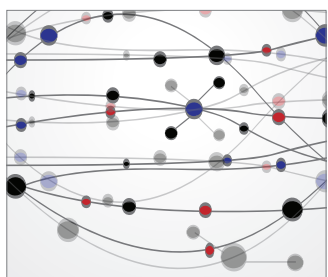

The Scientific World Journal
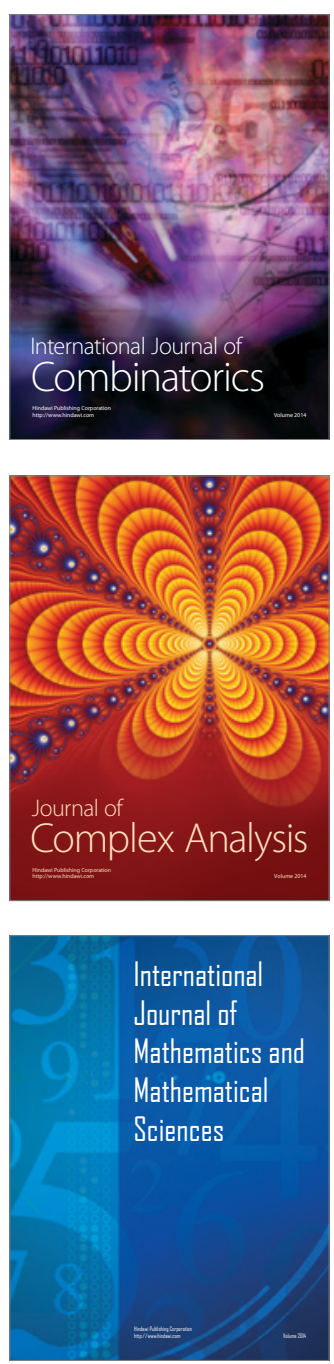
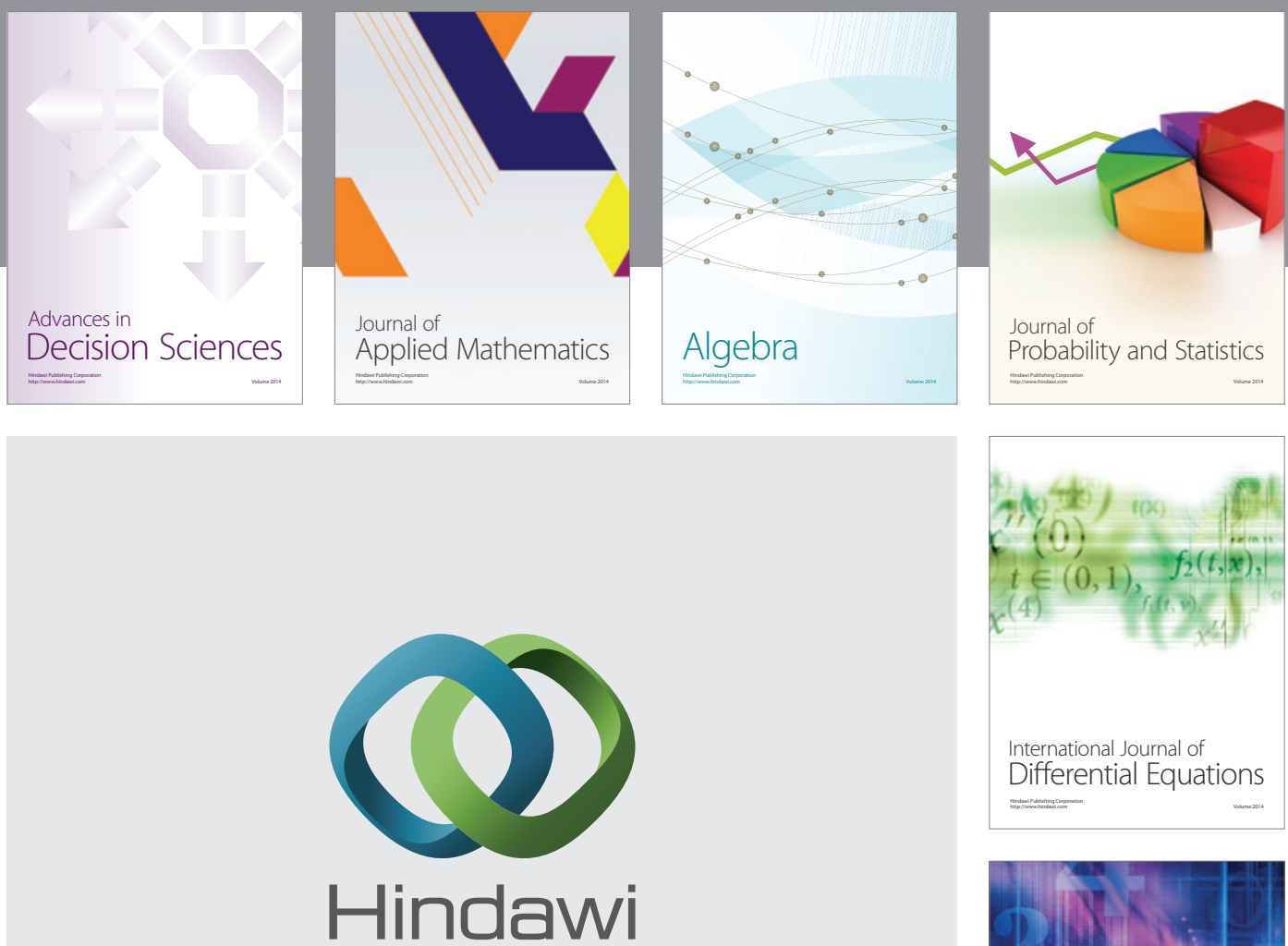

Submit your manuscripts at http://www.hindawi.com
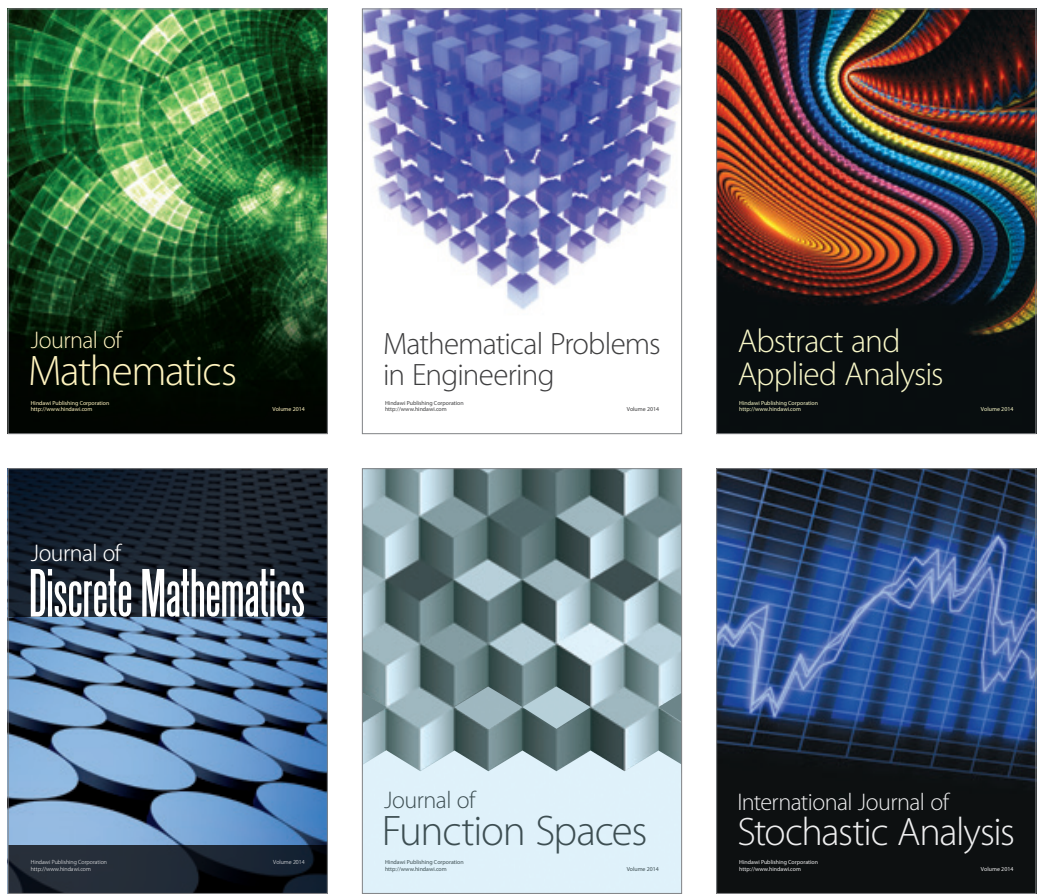

Journal of

Function Spaces

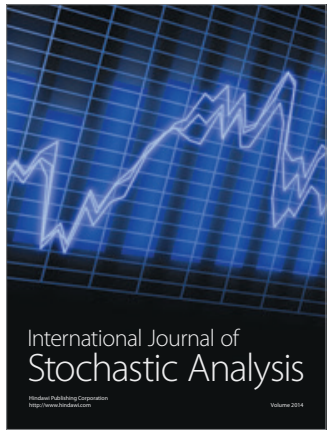

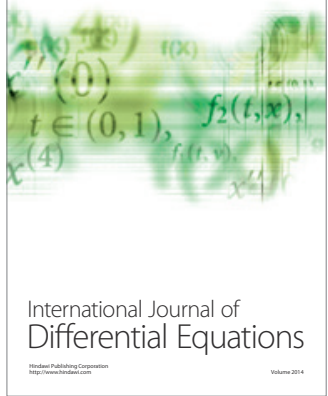
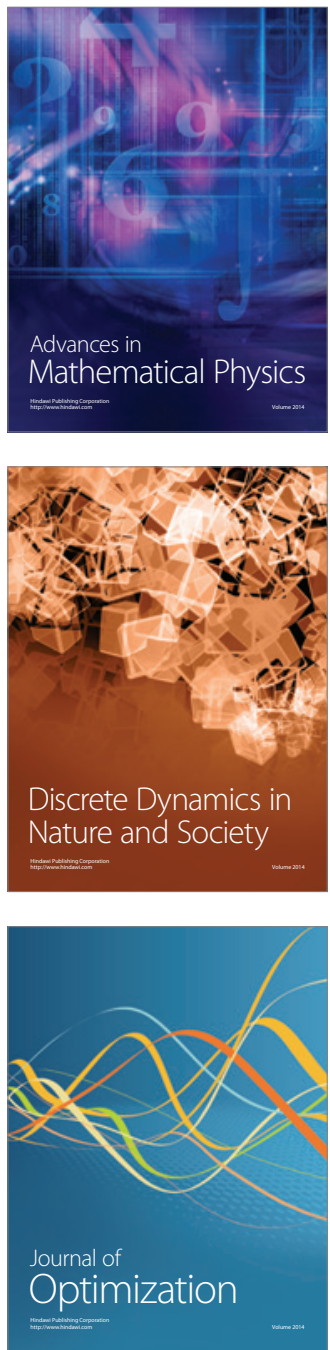